\title{
Evaluation of Molten Zone in Micro-welding of Glass by Picosecond Pulsed Laser
}

\author{
Yasuhiro Okamoto $^{* 1}$, Isamu Miyamoto $^{{ }^{*},{ }^{* 3}}$, Kristian Cvecek $^{* 4}$, \\ Akira Okada ${ }^{* 1}$, Kenta Takahashi ${ }^{* 1}$ and Michael Schmidt ${ }^{* 4,{ }^{*}}{ }^{*}$ \\ ${ }^{*}$ Graduate School of Natural Science and Technology, Okayama University, \\ 3-1-1 Thsushimanaka, Kita-ku, Okayama 700-8530, Japan \\ E-mail: okamoto@mech.okayama-u.ac.jp \\ ${ }^{*} 2$ Osaka University, 2-1, Yama-Oka, Suita, Osaka 565-0871, Japan \\ ${ }^{* 3}$ Erlangen Graduate School of Advanced Optical Technologies (SAOT), \\ Paul-Gordon Str. 6, 91052 Erlangen, Germany \\ ${ }^{*}$ Bayerisches Laserzentrum GmbH, Konrad-Zuse Str. 2-6, 91052 Erlangen, Germany \\ ${ }^{*}$ Friedrich-Alexander University Erlangen-Nuremberg, \\ Paul-Gordon Str. 6, 91052 Erlangen, Germany
}

\begin{abstract}
Glass materials have been widely used in optics, MEMS, electronics and biomedical applications because of their excellent optical, mechanical and chemical properties. Fusion welding by ultrashort pulsed laser has been proposed as a promising technique for glass joining, since joining can be accomplished without an intermediate layer and mechanical contact. However, to apply a fusion welding technique by ultrashort pulsed laser as a reliable joining method, it is important to understand the mechanical properties of the weld seam since glass materials are very sensitive to temperature gradients. Therefore, a method of evaluating the weld seam in the fusion welding of glass by picosecond pulsed laser was discussed, and its mechanical strength was investigated. A larger optical contact area had a great influence on the shear load of the weld seam, while a smaller optical contact area could reduce the influence of the contact area on the measurement of shear load. Therefore, it was important to reduce the optical contact area to accurately evaluate the shearing strength of weld seam. The mechanical strength of the weld seam reduced at a low pulse repetition rate. High bending strength and shear stress could be obtained at a proper pulse repetition rate.

DOI:10.2961/jlmn.2013.01.0013
\end{abstract}

Keywords: glass, micro-welding, ultrashort pulsed laser, picosecond laser, shear stress, bending strength, optical contact

\section{Introduction}

Glass materials have been widely used in the fields of optics, MEMS (Micro Electro Mechanical Systems), electronics and biomedical applications because of their excellent optical, mechanical, chemical properties and high thermal stability [1]. In general, an adhesive material is mainly used to join glass materials. The performance of this technique is greatly affected by the properties of the adhesive material and the processing environment. The joint accuracy might be affected by the heterogeneous shrinkage of the adhesive material, and the performance of the glass material such as mechanical strength and corrosion resistance may deteriorate. Therefore, a joining technique without an adhesive material or interlayer is required to overcome these problems [2]. The anodic bonding of glass to glass using an ultra-thin silicon interlayer has been proposed [3]. However, it is difficult to perform space selective joining using this technique.

An alternative approach is a direct joining technique, and a space selective joining method by an ultrashort pulsed laser based on multi-photon absorption has been proposed [4-6]. In addition, the fusion welding of glass with high pulse repetition rate by picosecond and femtosecond pulsed lasers has been reported [7-9], and the dou- ble-pulse train irradiation of a femtosecond pulsed laser could increase the efficiency in micro-welding of glass [10]. High-efficiency fusion welding is one of promising techniques for the space selective joining of glass without an intermediate layer. However, glass materials are brittle and very sensitive to temperature gradients. Since the glass material is locally heated up in this method, it is important to understand the mechanical properties of the weld part so that this fusion welding technique of glass by an ultrashort pulsed laser can be used as a reliable joining method. In addition, when a shearing test was carried out, it was confirmed that fracture occurred at the thin glass plate in the case of a large contact area between glass plates. This means the contact condition between glass plates makes it difficult to evaluate mechanical strengths correctly [11] Therefore, a method of evaluating the weld seam in the fusion welding of glass material by picosecond pulsed laser was investigated, and their mechanical strengths such as the bending and the shearing strength were discussed.

\section{Experimental setup for laser irradiation}

Picosecond pulsed laser (Time-Bandwidth, DUETTO) was used as the laser source, and the laser beam of 10ps pulse duration and $1064 \mathrm{~nm}$ wavelength was focused by an 


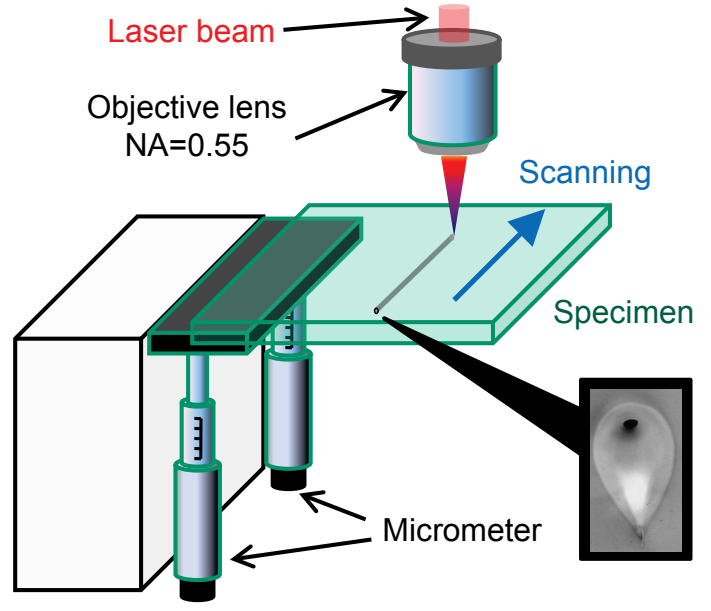

Fig. 1 Schematic illustration of laser irradiation experiment of glass plate.

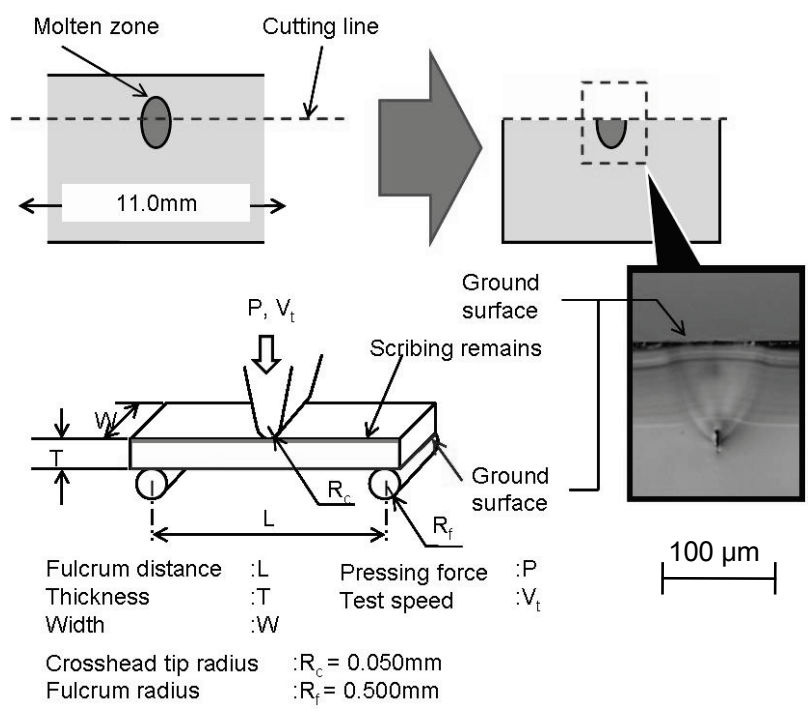

Fig. 2 Schematic illustration of three-point bending test.

objective lens of $0.55 \mathrm{NA}$. Fused silica and borosilicate glass (Schott, D263) of $1.1 \mathrm{~mm}$ thickness were used as a specimen, which was mounted on a motorized table as shown in Figure 1. Since the focusing depth had an influence on the weld seam [12], the specimen was aligned carefully to obtain the same focusing depth from the top surface and the interface between the glass plates. A weld seam was created by controlling the velocity of motorized table. After the creation of the weld seam, the specimens were cut, ground and polished perpendicularly to the irradiation line before evaluation by a bending test. The polished surfaces of all specimens were observed to confirm the shape of the molten zone. Before a shearing test, the welded area was measured from the top side, and the shearing test was carried out at various pulse repetition rates $R_{p}$ and feed rates $v$.

\section{Evaluation of molten zone by three-point bending} test

\subsection{Experimental procedure of three-point bending test}

A three-point bending test was carried out as shown in Figure 2. The test speed of crosshead and the fulcrum distance were $0.5 \mathrm{~mm} / \mathrm{min}$ and $10 \mathrm{~mm}$, respectively. A single line weld seam was generated in fused silica at conditions: scanning velocity $v=20 \mathrm{~mm} / \mathrm{s}$, average power $W_{i n}=6 \mathrm{~W}$, focusing depth from the top surface of specimen $\xi=-0.17 \mathrm{~mm}$. The laser irradiated specimen was cut into $1.5 \mathrm{~mm}$ width and more than $11 \mathrm{~mm}$ length. In order to divide into small size pieces correctly, the laser scribing was carried out. Qswitched YAG laser of $355 \mathrm{~nm}$ was used for scribing the bottom surface of specimen to avoid the crack generation at the molten zone. The top surface of separated specimen was ground and polished to the center of molten zone. The specimen was set with the polished surface facing downward. The scribed surface was faced upward so that cracks remaining on the scribed surface did not affect the measurement results of bending strength, because only compressive stress was applied in this scribed area.

\subsection{Three-point bending test results and discussion}

Figure 3 shows the results of the three-point bending test for various pulse repetition rates at scanning velocities of $20 \mathrm{~mm} / \mathrm{s}, 50 \mathrm{~mm} / \mathrm{s}$ and $200 \mathrm{~mm} / \mathrm{s}$. The light green areas indicate the range of bending strength measured from the original glass specimens which were not irradiated. Ten experiments were carried out for each set of experimental conditions, and the average and dispersion were evaluated.

In the case of low scanning velocity of $20 \mathrm{~mm} / \mathrm{s}$ as shown in Figure 3 (a), the bending strength equivalent to base material could be obtained at pulse repetition rates between $0.5 \mathrm{MHz}$ and $2.0 \mathrm{MHz}$. A molten zone was successfully created with only a small reduction of bending strength of approximately 30MPa compared with that of the non-irradiated specimens. On the other hand, there is a little reduction in the bending strength at pulse repetition rates of less than $0.3 \mathrm{MHz}$ and more than $3.0 \mathrm{MHz}$.

At the scanning velocity of $50 \mathrm{~mm} / \mathrm{s}$ as shown in Figure 3 (b), the bending strength of the molten zone equivalent to that of base material could be obtained at pulse repetition rate of less than $1.0 \mathrm{MHz}$, similar to the results at scanning velocity of $20 \mathrm{~mm} / \mathrm{s}$, while the bending strength decreased at pulse repetition rate above $2.0 \mathrm{MHz}$. The range of pulse repetition rates with high bending strength became narrower at the scanning velocity of $50 \mathrm{~mm} / \mathrm{s}$.

At the higher scanning velocity of $200 \mathrm{~mm} / \mathrm{s}$ as shown in Figure 3 (c), the bending strength of molten zone was lower than that of the base glass material for a wide range of pulse repetition rates, and the applicable pulse repetition rate to achieve an equivalent bending strength of approximately $130 \mathrm{MPa}$ was only $2.0 \mathrm{MHz}$. The bending strength at other pulse repetition rates was below $100 \mathrm{MPa}$.

The pulse repetition rate to achieve bending strength equivalent to base material became wider with decreasing the scanning velocity. The pulse energy decreased with increasing the pulse repetition rate under the same average power condition. However, the influence of the scanning velocity on bending strength was greater than that of the pulse repetition rate. The heat accumulation of laser energy had more effect on the bending strength. The low scanning velocity led to the gentle temperature change, while the high scanning velocity caused the rapid temperature change [13], which resulted in strong thermal stress. Therefore, the reduction of thermal stress is important to obtain the molten zone with higher bending strength, and it is considered that the setting of suitable processing parameters enables 


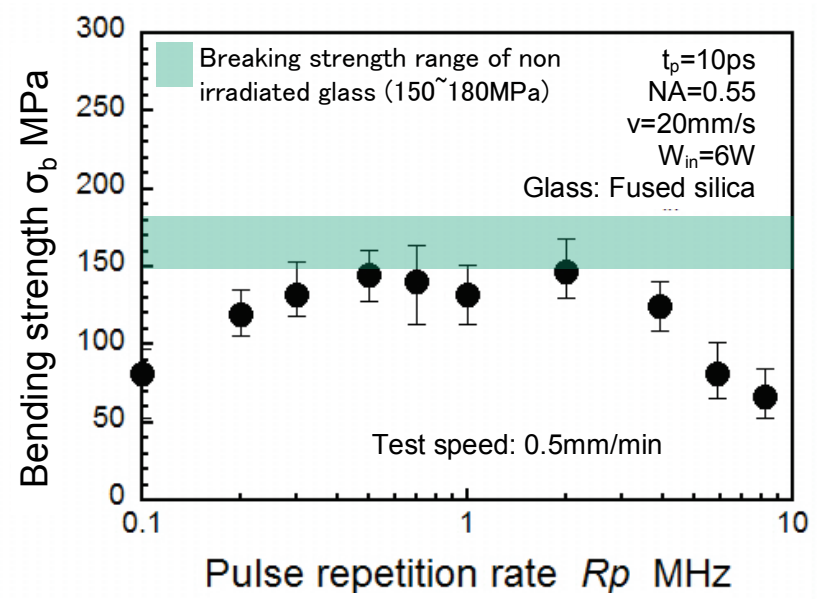

(a) Scanning velocity $v=20 \mathrm{~mm} / \mathrm{s}$

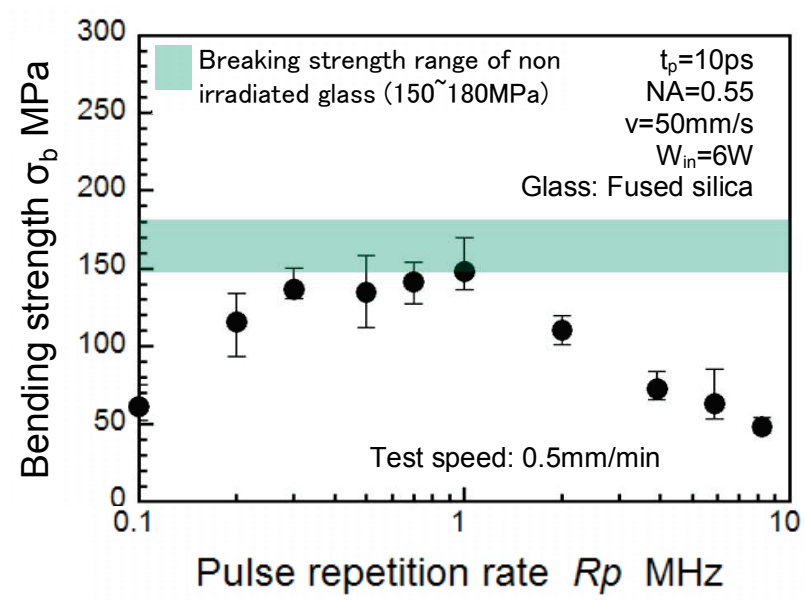

(b) Scanning velocity $v=50 \mathrm{~mm} / \mathrm{s}$

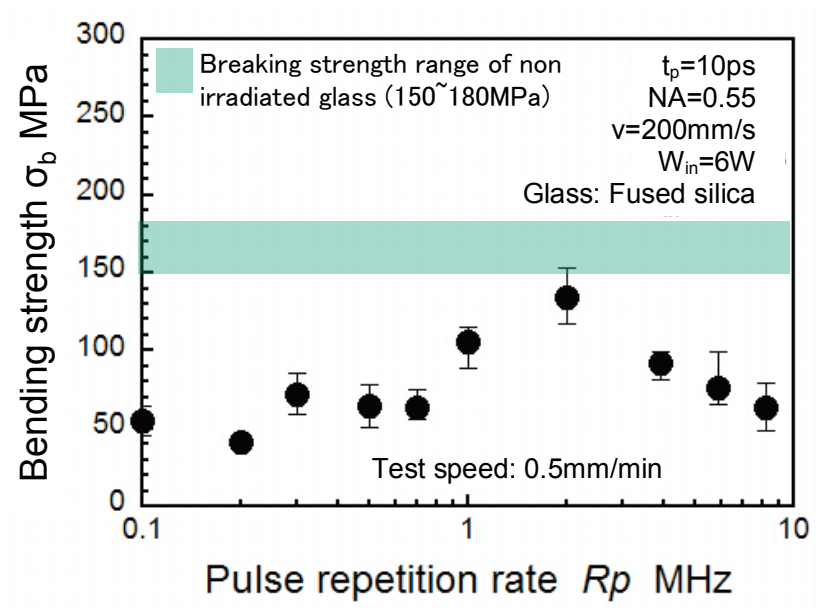

(c) Scanning velocity $\mathrm{v}=200 \mathrm{~mm} / \mathrm{s}$

Fig. 3 Bending strength of molten zone by three-point bending test.

the highly reliable fusion welding of glass without a fatal reduction of mechanical strength.

\section{Evaluation by shearing test}

\subsection{Experimental procedure of shearing test}

If the interface between the glass plates retains a good contact condition over a large area, its contact condition had a great influence on the measurement results of the

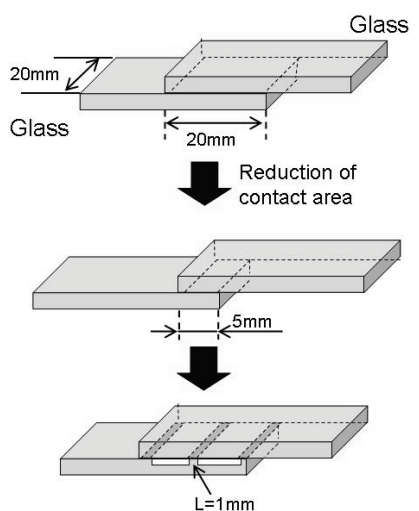

(a) Setup of specimen

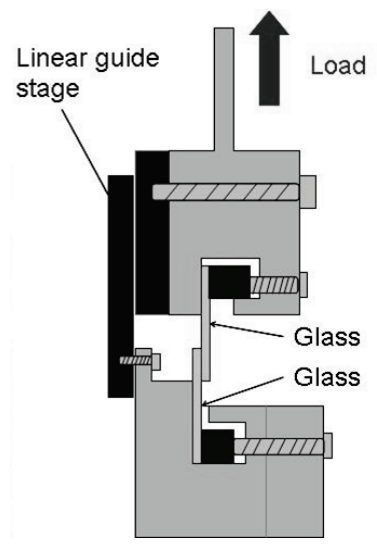

(b) Clamping device
Fig.4 Schematic illustration of specimen setup and clamping device for shearing test.

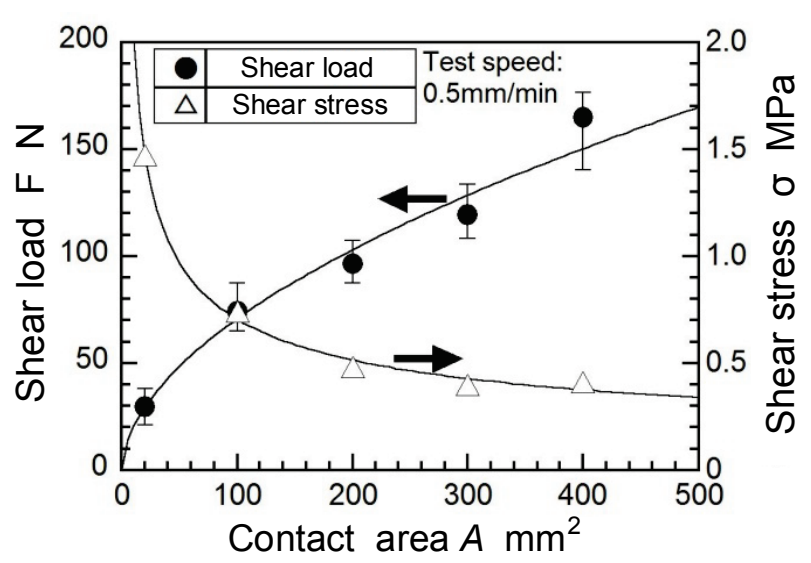

Fig.5 Shear load and stress to break optical contact for various contact areas.

shearing test [11]. In order to discuss the optical contact force, various optical contact areas between two borosilicate glass plates D263 of $1.1 \mathrm{~mm}$ thickness were prepared by changing the contact length $L$ with a constant width of $20 \mathrm{~mm}$ as shown in Figure 4 (a). The optical contact is marked by a lack of light reflection from the interface between the glass plates. The smaller contact length $L=1 \mathrm{~mm}$ was produced by HF etching of masked glass specimen. Two glass plates were placed together immediately after cleaning with an ethanol as specimens for the shearing test. A shearing test of these specimens were performed by using a clamping device with a linear guide in order to prevent them from twisting during the pulling process as shown in Figure 4 (b). The friction force of the linear guide was subtracted from the maximum load, and the shear stress was calculated from the measured area of the weld seam. Ten experiments were carried out for each set of experimental conditions, and the average and dispersion were evaluated.

\subsection{Influence of optical contact area}

The shear load to break the optical contact area was evaluated by the specimen of two glass plates fixed by the optical contact condition without the weld seam [14]. The shear load to break the optical contact area was obtained as shown in Figure 5. The shear load increased with in- 


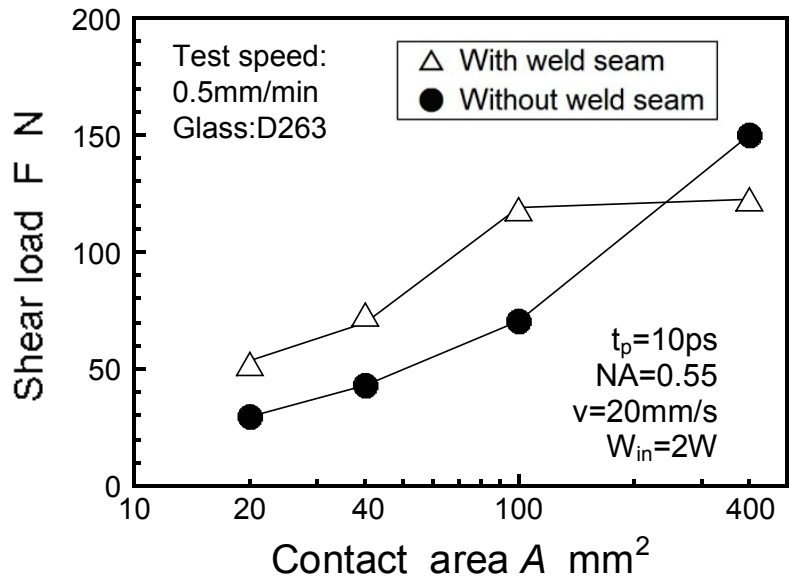

Fig.6 Influence of optical contact area on shear load.

creasing contact area, while the shear stress increased with decreasing contact area. A large contact area led to a lack of uniformity of the contact condition. On the other hand, a small contact area could improve the uniformity of the contact condition, and thus it is considered that a higher shear stress was obtained in the case of a small contact area. The combination of a large contact area and a small weld seam should be noticed, since a high shear load might be measured due to the optical contact force. Moreover, the dispersion of the shear load decreased with decreasing contact area, and a smaller contact area led to the more stable measurement of the shear load.

The laser irradiation experiments were carried out at the interface between the two glass plates D263, and one weld seam of $15 \mathrm{~mm}$ length was obtained in $20 \mathrm{~mm}$ contact width specimen for various contact areas. The weld seam was located at the center of the contact length $L$. Since the location of the weld seam in the optical contact area might affect the measurement results, its location was carefully controlled. The focusing depth was controlled as the center of molten zone located at the interface between the two glass plates. A lower average power was applied to borosilicate glass D263 compared with the fused silica, since the molten area of D263 is approximately four times larger than that of fused silica due to its lower melting temperature and thermal diffusivity [15].

Figure 6 shows the shear loads with and without the weld seam for various contact areas under the same laser irradiation condition. A weld seam without a fatal defect was obtained under this irradiation condition as shown in the figure. The shear load of the glass plate fixed by the optical contact force without the weld seam increased with increasing contact area, and these values were smaller than those with a weld seam at contact area from 20 to $100 \mathrm{~mm}^{2}$. However, at a large contact area $400 \mathrm{~mm}^{2}$, the shear load with the weld seam was conversely smaller than that with only an optical contact force. The larger contact area greatly affected the shear load of the weld seam. It is considered that the generation of the weld seam changed the contact condition between the glass plates, and the loose contact might decrease the shear load. On the other hand, at the smaller contact area, the shear load with the weld seam became larger than that without the weld seam, and it is considered that the value subtracted the optical contact

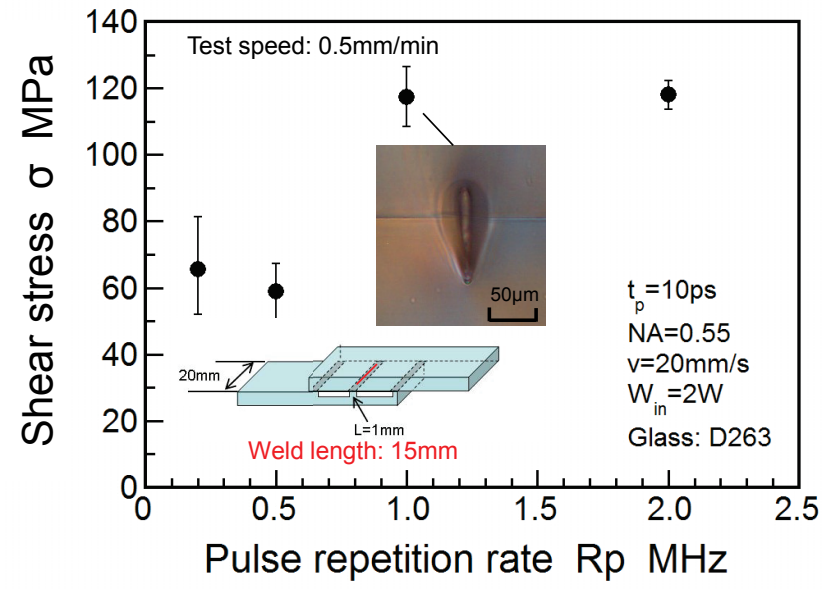

Fig.7 Influence of pulse repetition rate on shear stress.

force from the total shear load was appropriate evaluation as the shear load of the weld seam [14]. However, the difference in shear load with and without the weld seam slightly decreased with decreasing contact area, and appeared to approach a constant value with decreasing the contact area. Although further experiments are needed to quantify this analysis, it is considered that a smaller contact area is important for achieving the more reliable evaluation of shear load in the micro-welding of glass plates.

\subsection{Shearing test results and discussion}

As mentioned above, in the case of a large optical contact area, the shear load to break the optical contact was larger than that for the weld seam, and the measurement value of shear load was related to the optical contact conditions such as loose or tightened contact by the creation of the weld seam. On the other hand, in the case of a smaller contact area, the shear load to break the optical contact became smaller, and the reduction of optical contact area was essential to reduce the influence of optical contact force on the measurement of shear load for the reliable evaluation of the weld seam strength.

The shear stress of a weld seam was measured by using smaller contact areas $20 \mathrm{~mm}^{2}$. The maximum shear load was divided by the area of the weld seam, and its value was plotted as the shear stress in Figure 7. It was clarified that the pulse repetition rate had a great influence on the shear stress, which decreased at the lower pulse repetition rate below $0.5 \mathrm{MHz}$. On the other hand, a higher shear stress could be obtained at a pulse repetition rate more than 1.0MHz. The reduction of the optical contact area led to the reliable evaluation of shear stress, and a stronger weld seam could be achieved under the higher pulse repetition rate condition.

Figure 8 shows SEM (Scanning Electron Microscope) photographs of fracture faces after the shearing test. At a low pulse repetition rate with the low shear load, molten zone was peeled off largely. On the other hand, at a higher pulse repetition rate with a high shear stress, molten zone was peeled off with non-molten zone, which indicated that strong joining could be achieved. As shown in Figure 3, a higher bending strength could be achieved at a high pulse repetition rate more than $1.0 \mathrm{MHz}$ at all scanning velocities, and the pulse repetition rate was strongly related to the 

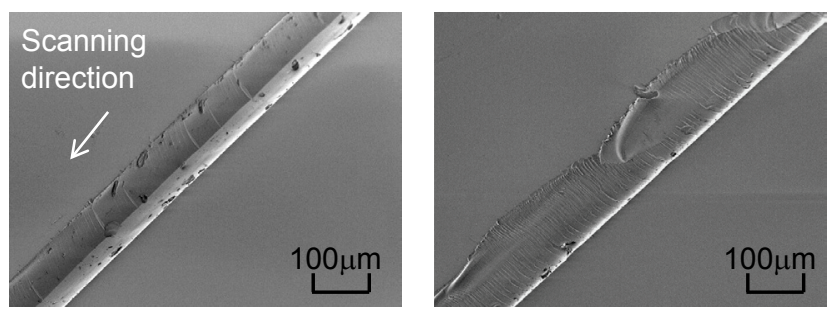

$t_{p}=10 p s, N A=0.55, v=20 \mathrm{~mm} / \mathrm{s}, W_{\text {in }}=2 \mathrm{~W}$, Glass: $D 263$

(a) $\mathrm{R}_{\mathrm{p}}=0.5 \mathrm{MHz}$

(b) $\mathrm{R}_{\mathrm{p}}=1.0 \mathrm{MHz}$

Fig.8 Fracture faces after shearing test

change of temperature. The pulse energy was varied by changing the pulse repetition rate under the same average power condition. The change of temperature became greater at a lower pulse repetition rate with higher pulse energy. Its thermal load led to large variations of internal stress, which had an influence on the mechanical properties of the weld seam and its surrounding area. On the other hand, a high pulse repetition rate with lower pulse energy led to smaller variations of internal stress compared with the low pulse repetition rate. Therefore, the pulse repetition rate has an influence on the mechanical strength of weld seam, and the higher mechanical strength could be achieved under the proper pulse repetition rate with a constant average input power condition.

\section{Conclusions}

In this study, a method evaluating the mechanical strength of the weld seam in fusion welding of glass material by a picosecond pulsed laser was investigated, and the bending strength and shearing stress were discussed. The main conclusions obtained in this study are as follows:

(1) A higher bending strength could be achieved at the wider range of pulse repetition rates in the case of a low scanning velocity.

(2) The optical contact condition of the interface between glass plates had a great influence on the shear load, and the reduction of the optical contact area was important for reliable evaluation of the shear stress of a weld seam in fusion welding of glass.

(3) The mechanical strength was lower at the low pulse repetition rate, and a high bending strength and shear stress could be achieved under the proper pulse repetition rate condition.

\section{Acknowledgments}

The authors would like to thank Prof. Dr. Yoshiharu Namba, Chubu University for helpful discussion. This work was partially supported by Grant-in-Aid for Young Scientists (B) (No. 21760100), Japan Society of the Promotion of Science.

\section{References}

[1] Y. Zhou: "Microjoining and Nanojoining" (Woodhead publishing, 2008) p.356.

[2] T. Yoshida: "High Performance Glass and Nanoglass Advanced Technology" (NTS 2006) p.80. (In Japanese)

[3] D. J. Lee, B. Ju, Y. Lee, J. Jang and M. Oh: Glass-toGlass Anodic Bonding for High Vacuum Packaging of
Microelectronics and its Stability, Proc. of IEEE The Thirteenth Annual International Conference on Micro Electro Mechanical System, (2000) 253.

[4] T. Tamaki, W. Watanabe, J. Nishii and K. Itoh: Welding of Transparent Materials Using Femtosecond Laser Pulses, Japanese Journal of Applied Physics, Vol.44, No.22, (2005) 687.

[5] T. Tamaki, W. Watanabe and K. Itoh: Laser Microwelding of Transparent Materials by A Localized Heat Accumulation Effect Using A Femtosecond Fiber Laser at $1558 \mathrm{~nm}$, Optics Express, Vol.14, No.22, (2006) 10460 .

[6] W. Watanabe, S. Onda, T. Tamaki, K. Itoh and J. Nishii: Space-selective Laser Joining of Dissimilar Transparent Materials Using Femtosecond Laser Pulses, Applied Physics Letters, Vol.89, No.2, (2006) 021106.

[7] I. Miyamoto, A. Horn and J. Gottomann: Local Melting of Glass Material and Its Application to Direct Fusion Welding by Ps-laser Pulses, Journal of Laser Micro/Nanoengineering, Vol.2, No.1, (2007) 7.

[8] F. Yoshino, J. Bovatsek, A. Arai, Y. Uehara, Z. Liu and G. Cho: High Energy-High Repetition Rate Fiber Laser System for Precision Micromachining with Fundamental and Second Harmonic Wavelengths, Journal of Laser Micro/Nanoengineering Vol.1, No.3, (2006) 258.

[9] M. L. Stock, L. Shah, B. Liu, M. Yoshida, F. Yoshino, J. Bovatsek and A. Arai: Optimized precision micromachining using commercially available, highrepetition rate microjoule femtosecond fiber lasers, Proceedings of the Society of Photo-optical Instrumentation Engineers (SPIE), Vol.6108, article number: 61080Q (2006).

[10] K. Sugioka, M. Iida, H. Takai and K. Midorikawa: Efficient Microwelding of Glass Substrates by Ultrafast Laser Irradiation Using A Double-pulse Train, Optics Letters, Vol.36, (2011) 2734.

[11] K. Cvecek, I. Miyamoto, J. Strauss, M. Wolf, T. Frick and M. Schmidt: Sample Preparation Method for Glass Welding by Ultrashort Laser Pulses Yields Higher Seam Strength, Applied Optics, Vol.50, No.13, (2011) 1941.

[12] K. Cvecek, I. Miyamoto, M. Rascher, T. Frick and M. Schmidt: Shear Measurements of Joining Seams in Glass Welded by Ultra-fast Lasers Depending on Focus Height, Proceedings of The 12th International Symposium on Laser Precision Microfabrication, (2011) On-line.

[13] I. Miyamoto and T. Herrmann: Fusion Welding of Fused Silica by Ultrashort Pulse Laser, Proceedings of The 9th International Symposium on Laser Precision Microfabrication, (2011) On-line.

[14] I. Miyamoto, K. Cvecek, Y. Okamoto, M. Schmidt and H. Helvajian: Characteristics of Laser Absorption and Welding in FOTURAN Glass by Ultrashort Laser Pulses, Optics Express, Vol.19, No.23, (2011) 22961.

[15] I. Miyamoto, K. Cvecek, Y. Okamoto and M. Schmidt: Novel Fusion Welding Technology of Glass Using Ultrashort Pulse Lasers, Physics Procedia, Vol.5 (2010) 483.

(Received: June29, 2012, Accepted: January 5, 2013) 